\title{
Erratum to: Fine mapping and candidate gene analysis of $q H D 5$, a novel major QTL with pleiotropism for yield-related traits in rice (Oryza sativa L.)
}

\author{
Bin Sun ${ }^{1,2}$ Xiao-Deng Zhan ${ }^{1,2} \cdot$ Ze-Chuan Lin ${ }^{1,2} \cdot$ Wei-Xun Wu ${ }^{1,2} \cdot$ Ping Yu ${ }^{1,2}$. \\ Ying-Xin Zhang ${ }^{1,2} \cdot$ Lian-Ping Sun ${ }^{1,2} \cdot$ Li-Yong Cao ${ }^{1,2} \cdot$ Shi-Hua Cheng ${ }^{1,2}$
}

Published online: 29 November 2016

(C) Springer-Verlag Berlin Heidelberg 2016

\section{Erratum to: Theor Appl Genet DOI 10.1007/s00122-016-2787-y}

Unfortunately, there was a mistake in "Key message" (abstract section) of the original publication. The text should read "52.59-kb".

The complete corrected sentence is given below:

Key message A major QTL for heading date, $q H D 5$, was fine-mapped to a $52.59-\mathrm{kb}$ region on the short arm of rice chromosome 5 .

The original article was corrected.

The online version of the original article can be found under doi:10.1007/s00122-016-2787-y.

Li-Yong Cao

caolycgf@mail.hz.zj.cn

$\triangle$ Shi-Hua Cheng

shcheng@mail.hz.zj.cn

1 Chinese National Center for Rice Improvement, China National Rice Research Institute, Hangzhou 310006, China

2 State Key Laboratory of Rice Biology, China National Rice Research Institute, Hangzhou 310006, China 